\title{
Neoadjuvante Chemoradiotherapie bei Ösophagustumoren
}

\author{
Patienten mit Adenokarzinom von Speiseröhre oder gastroösophagealem \\ Übergang profitieren hinsichtlich des Gesamtüberleben, wenn sie vor der \\ Resektion eine neodajuvante Chemotherapie oder Chemoradiotherapie \\ durchlaufen. In einer in einer schwedisch-norwegischen Studie wurde nun der \\ Effekt beider Regimes auf das pathologische Ansprechen evaluiert.
}

$B^{c}$ ei Tumoren des Ösophagus und gastroösophagealen Übergangs wird ein histologisch vollständiges Ansprechen nach neoadjavanter Therapie häufig als starker und relevanter Prädiktor für einen günstigen Verlauf gedeutet. In einer randomisierten Phase-II-Studie wurde daher die Rate an histologischen Komplettremissionen nach neoadjuvanter Chemotherapie mit der nach neoadjuvanter Chemoradiotherapie verglichen.

Sekundäre Endpunkte waren Gesamtüberleben (OS), die Rate an R0-Resektionen, das progressionsfreie Überleben und die Lokalisation auftretender Rezidive.

181 Patienten erhielten randomisiert eine neoadjuvante Chemotherapie (3
Zyklen Cisplatin-/5-Fluorouracil [5FU] q3w) mit Bestrahlung (nCRT; 40 Gy in 20 Fraktionen) oder ohne (nCT), jeweils gefolgt von einer chirurgischen Tumorresektion mit 2-Feld-Lymphadenektomie.

$28 \%$ der Patienten im nCRT- und 9\% im nCT-Arm erreichten eine histologische Komplettremission ( $p=0,002)$. Vorteile für die mit nCRT vorbehandelte Gruppe ergaben sich auch im Hinblick auf die R0-Resektionsrate: Diese betrug $87 \%$ unter nCRT im Vergleich zu $74 \%$ unter nCT ( $\mathrm{p}=0,04)$. $62 \%$ der resezierten Patienten im nCT-Arm, aber lediglich $32 \%$ im nCRT-Arm entwickelten Lymphknotenmetastasen $(p=0,001)$.
Beide Behandlungsarme unterschieden sich nicht, was das 3-Jahres-OS anging: $49 \%$ im nCT- und $47 \%$ im nCRT-Arm $(\mathrm{p}=0,77)$.

Fazit: Bei Patienten mit einem Karzinom des Ösophagus oder gastroösophagealen Übergangs erhöht die um eine Strahlenbehandlung erweiterte neoadjuvante Chemotherapie die Rate für das komplette histologische Ansprechen, verbessert die Chance auf eine R0-Resektion und verringert die Frequenz von Lymphknotenmetastasen. Diese Vorteile bleiben jedoch ohne Einfluss auf das OS. Komplettes histologisches Ansprechen stellt somit keinen Surrogatmarker für das Überleben dar, resümieren die Forscher.

\section{Wolfgang Zimmermann}

Klevebro $\mathrm{F}$ et al. A randomized clinical trial of neoadjuvant chemotherapy versus neoadjuvant chemoradiotherapy for cancer of the oesophagus or gastro-oesophageal junction. Ann Oncol. 2016;27(4):660-7.

\section{„Austherapiertes" Magenkarzinom: Angiogenesehemmer als Alternative?}

\begin{abstract}
Bis dato existieren keine standardisierten Therapieempfehlungen für Patienten mit einem chemotherapierefraktären fortgeschrittenen Adenokarzinom des Magens oder gastroösophagealen Übergangs. Chinesische Mediziner untersuchten in einer Phase-III-Studie Wirksamkeit und Sicherheit eines neuen oralen Angiogeneseinhibitors bei diesen Patienten.
\end{abstract}

siert im Verhältnis 2:1 wurden die Patienten entweder mit Apatinib (850 mg pro Tag) in 4-wöchigen Zyklen oder ein entsprechendes Placebopräparat. Die Patienten setzten die Therapiezyklen bis zur Krankheitsprogression oder dem Auftreten intolerabler Nebenwirkungen fort.

In Übereinstimmung mit den Ergebnissen der Phase-II-Studie zeigte sich unter der Apatinib-Behandlung ein signifikant besseres OS und PFS im Vergleich zur Placebogruppe: median 6,5 versus 4,7 Monate $(p=0,0149)$ bzw. median 2,6 versus 1,8 Monate $(\mathrm{p}=0,0156)$. Als häufigste nichthämatologische Nebenwirkungen traten Hand-Fuß-Syn- drom, Hypertonie und Proteinurämie auf, erwartungsgemäß gehäuft unter Apatinib. Im Vergleich mit anderen Angiogenesehemmern beurteilen die Forscher das Nebenwirkungsprofil von Apatinib als eher günstig.

Fazit: Nach diesen Ergebnissen verbessert der Tyrosinkinaseinhibitor Apatinib als Monotherapie signifikant OS und PFS von erfolglos chemotherapeutisch vorbehandelten Patienten mit fortgeschrittenem Karzinom des Magens oder gastroösophagealen Übergangs. Als Herausforderung bleibt die Identifizierung von Patienten, die am besten auf die Therapie mit Angiogeneseinhibitoren ansprechen.

Wolfgang Zimmermann

Li J et al. Randomized, double-blind, placebo-controlled phase III trial of apatinib in patients with chemotherapy-refractory advanced or metastatic adenocarcinoma of the stomach or gastroesophageal junction. J Clin Oncol. 2016;34(13):1448-54. 